\title{
Endoscopic Ultrasound (EUS)-Directed Transgastric Endoscopic Retrograde Cholangiopancreatography or EUS: Mid-Term Analysis of an Emerging Procedure
}

\author{
Amy Tyberg ${ }^{1}$, Jose Nieto ${ }^{2}$, Sanjay Salgado ${ }^{1}$, Kristen Weaver ${ }^{1}$, Prashant Kedia ${ }^{3}$, Reem Z. Sharaiha ${ }^{1}$, Monica Gaidhane ${ }^{1}$ and Michel \\ Kahaleh $^{1}$ \\ ${ }^{1}$ Division of Gastroenterology and Hepatology, Weill Cornell Medical Center, New York, NY, ${ }^{2}$ Division of Gastroenterology and Hepatology, \\ Borland-Groover Clinic (BGC), Jacksonville, FL, ${ }^{3}$ Interventional Endoscopy, Methodist Dallas Medical Center, Dallas, TX, USA
}

Background/Aims: Performing endoscopic retrograde cholangiopancreatography (ERCP) in patients who have undergone Rouxen-Y gastric bypass (RYGB) is challenging. Standard ERCP and enteroscopy-assisted ERCP are associated with limited success rates. Laparoscopy- or laparotomy-assisted ERCP yields improved efficacy rates, but with higher complication rates and costs. We present the first multicenter experience regarding the efficacy and safety of endoscopic ultrasound (EUS)-directed transgastric ERCP (EDGE) or EUS.

Methods: All patients who underwent EDGE at two academic centers were included. Clinical success was defined as successful ERCP and/or EUS through the use of lumen-apposing metal stents (LAMS). Adverse events related to EDGE were separated from ERCPor EUS-related complications and were defined as bleeding, stent migration, perforation, and infection.

Results: Sixteen patients were included in the study. Technical success was $100 \%$. Clinical success was $90 \%$ ( $n=10)$; five patients were awaiting maturation of the fistula tract prior to ERCP or EUS, and one patient had an aborted ERCP due to perforation. One perforation occurred, which was managed endoscopically. Three patients experienced stent dislodgement; all stents were successfully repositioned or bridged with a second stent. Ten patients (62.5\%) had their LAMS removed. The average weight change from LAMS insertion to removal was negative $2.85 \mathrm{~kg}$.

Conclusions: EDGE is an effective, minimally invasive, single-team solution to the difficulties associated with ERCP in patients with RYGB. Clin Endosc 2017;50:185-190

Key Words: EUS-directed transgastric ERCP; Lumen-apposing metal stent; Cholangiopancreatography, endoscopic retrograde; Gastric bypass

\section{INTRODUCTION}

The obesity epidemic, coupled with the relative failure of non-invasive weight loss reduction techniques, has led to the growth of bariatric surgery. Between 1998 and 2008, the United States witnessed a 10-fold increase in bariatric surgeries. ${ }^{1,2}$

Received: February 3, 2016 Accepted: June 27, 2016

Correspondence: Michel Kahaleh

Division of Gastroenterology and Hepatology, Weill Cornell Medical College, 1305 York Avenue, 4th floor, New York, NY 10021, USA

Tel: +1-646-962-4000, Fax: +1-646-962-0110, E-mail: mkahaleh@gmail.com

cc This is an Open Access article distributed under the terms of the Creative Commons Attribution Non-Commercial License (http://creativecommons.org/ licenses/by-nc/3.0) which permits unrestricted non-commercial use, distribution, and reproduction in any medium, provided the original work is properly cited.
With a growing body of evidence expounding the value of bariatric surgery in controlling obesity and its complications, ${ }^{3-6}$ there is a growing need to develop diagnostic and therapeutic techniques that are effective for patients with the altered anatomy that results from these procedures.

Roux-en-Y gastric bypass (RYGB) is the most commonly performed bariatric operation. Patients who have undergone RYGB pose unique challenges in situations wherein endoscopic retrograde cholangiopancreatography (ERCP) is required. These difficulties include maneuvering through gastrointestinal anastomoses as well as identifying and gaining access to the ampulla of Vater and the biliary tree while approaching from a reverse direction without a side-viewing scope or other 
common ERCP accessories.

A number of transoral and surgical approaches have been described for ERCP in these patients. Transoral approaches include the use of standard ERCP, which has been shown to have success rates as low as $33 \%$ in patients with RYGB, ${ }^{8}$ and enteroscopy-assisted ERCP (EA-ERCP), ${ }^{9}$ which has suboptimal success rates of $70 \%{ }^{10}$ secondary to the lack of an elevator and forward vision on the enteroscope. Surgical approaches consist of laparoscopy- or laparotomy-assisted ERCP through a gastrostomy tract (LA-ERCP), ${ }^{11,12}$ which carries impressive technical success rates compared to EA-ERCP (95\% vs. 63\%), but is associated with higher complication rates (14.5\% vs. $3.1 \%)$, as well as higher healthcare costs and difficulties with coordination between multiple interdisciplinary teams. ${ }^{13-16}$

Recently, an endoscopic ultrasound (EUS)-guided procedure for ampullary access has been developed, termed internal EUS-directed transgastric ERCP (EDGE). This technique involves accessing the excluded stomach from the gastric pouch by placing a lumen-apposing metal stent (LAMS) across a fistula tract with EUS guidance, and subsequently performing conventional ERCP through the LAMS. ${ }^{17,18}$ We present the first multicenter experience regarding the efficacy and safety of the EDGE technique.

\section{MATERIALS AND METHODS}

\section{Study overview}

All patients who underwent EDGE between March 2014 and August 2015 in two academic centers in the United States were included in a prospective registry. Demographic data, procedural information, and clinical follow-up data were documented. Technical success was defined as successful deployment of the LAMS. Clinical success was defined as suc-

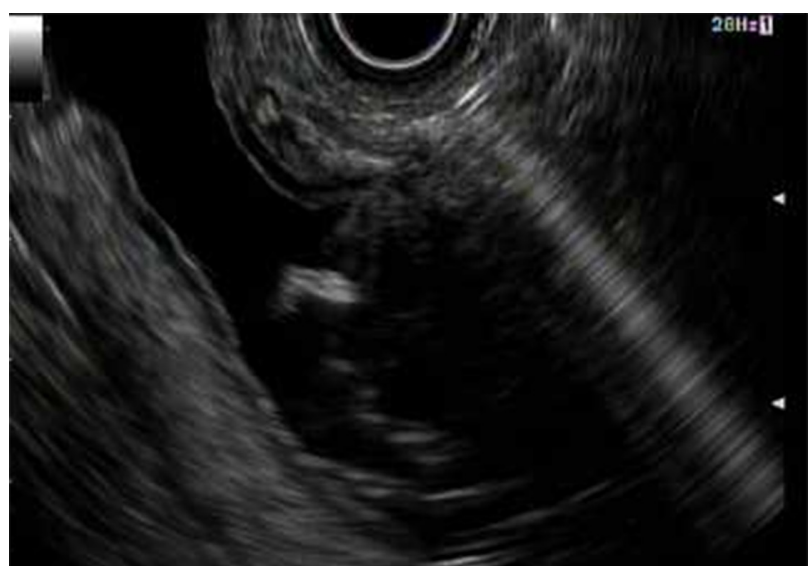

Fig. 1. Endoscopic ultrasound image of endoscopic ultrasound-fine needle aspiration of bypassed stomach. cessful ERCP and/or EUS through the LAMS. Adverse events related to EDGE were separated from ERCP- or EUS-related complications, and were defined as procedural or postprocedural bleeding, stent migration, perforation, and infection. All patients provided written informed consent prior to the procedure.

\section{Procedural technique}

The excluded stomach was located endosonographically with a linear echoendoscope (GF-UCT180; Olympus, Central Valley, PA, USA) from the remnant gastric pouch or the afferent limb, and then accessed with a 19-gauge EUS needle (ECHO-19; Cook Medical, Winston-Salem, NC, USA) (Fig. 1).

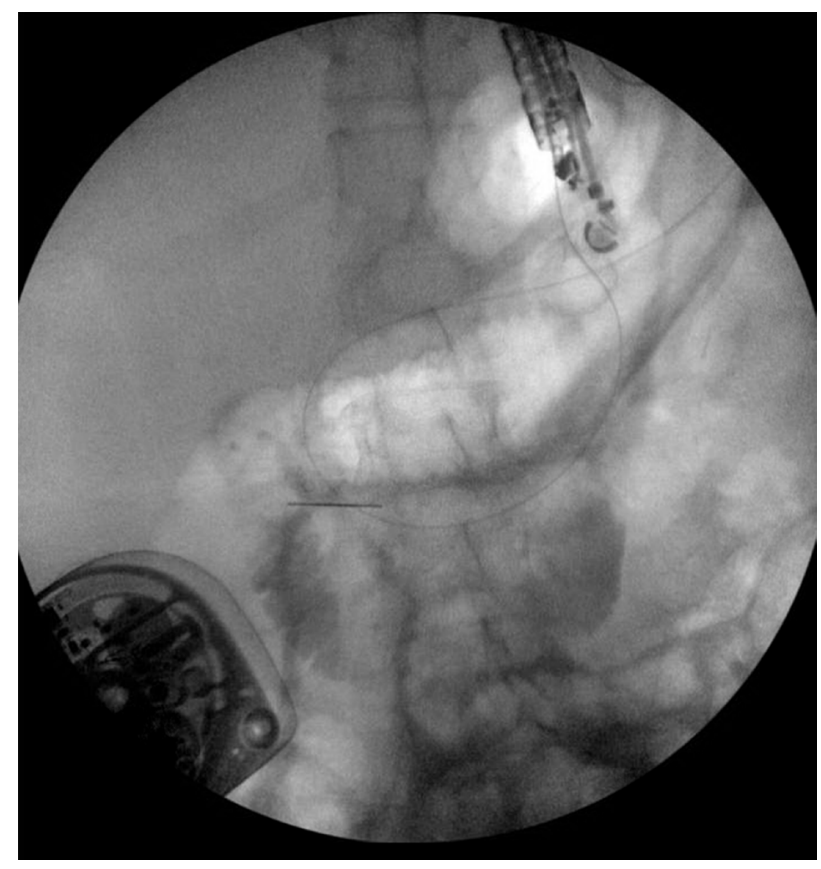

Fig. 2. Fluoroscopic image of coiling of the wire within the bypassed stomach.

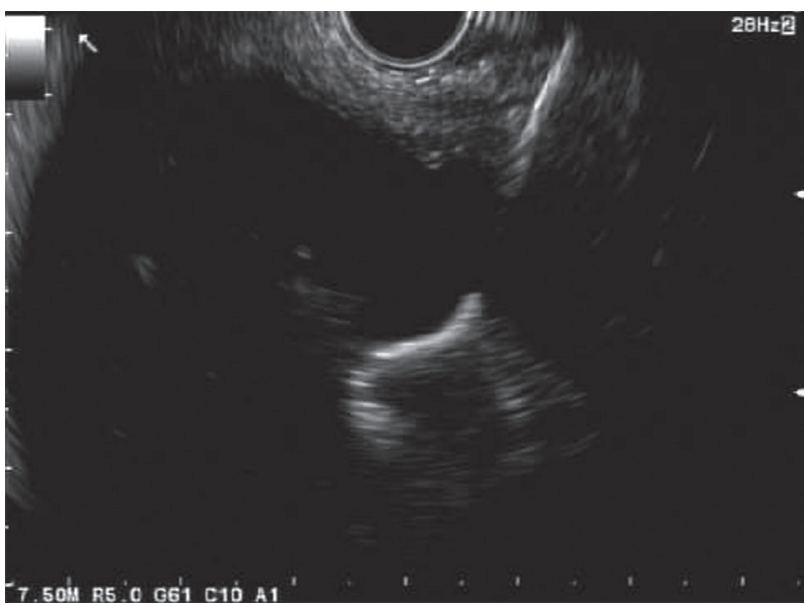

Fig. 3. Endoscopic ultrasound image of deployment of the lumen-apposing metal stent. 


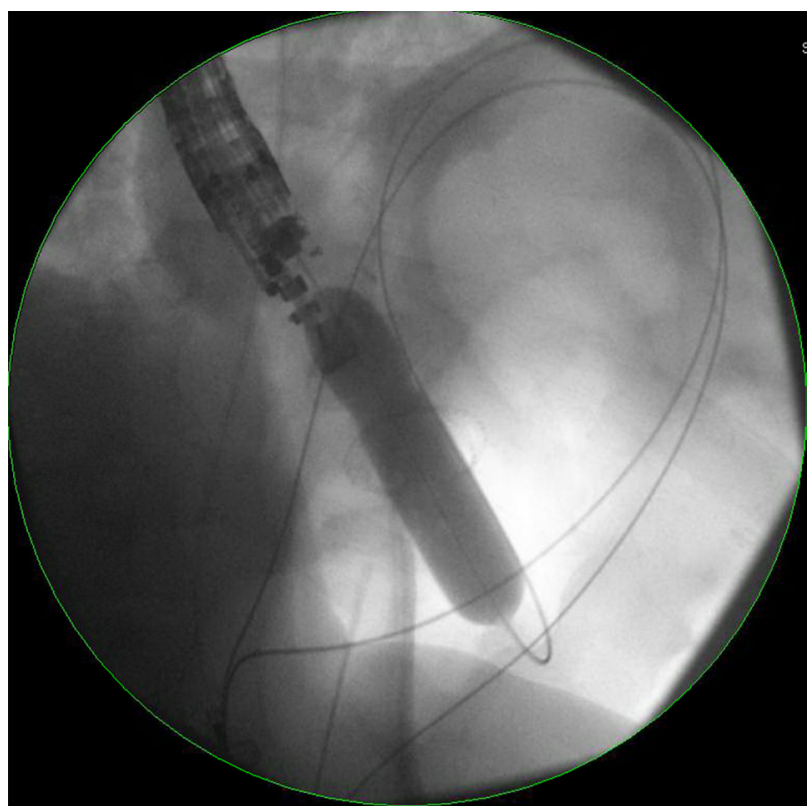

Fig. 4. Fluoroscopic image of balloon dilation of the lumen of the lumen-apposing metal stent.

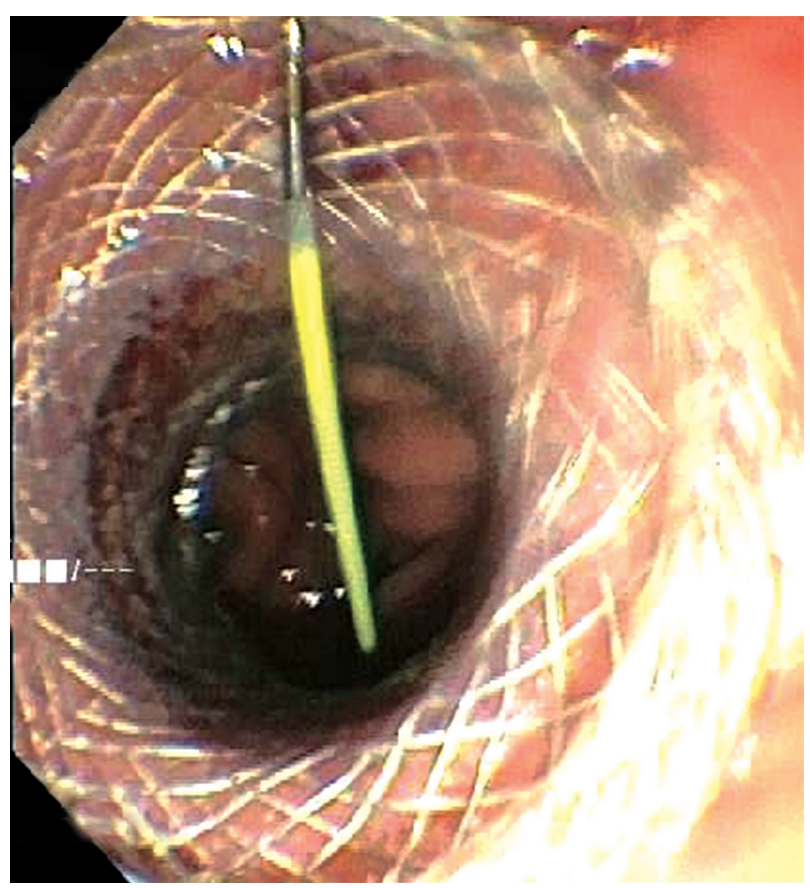

Fig. 5. Endoscopic view of the lumen-apposing metal stent after deployment.

Contrast, along with $120 \mathrm{cc}$ of water, was injected through the 19-gauge needle to confirm the position within and distend the excluded stomach. A 0.035" wire (HydraJag; Boston Scientific, Natick, MA, USA) was advanced through the needle and coiled within the lumen of the excluded stomach (Fig. 2). The fistula tract was created with cautery (RX Needle Knife; Boston Scientific) and dilated with a $4 \mathrm{~mm}$ balloon (Hurricane RX; Boston Scientific) prior to advancement of

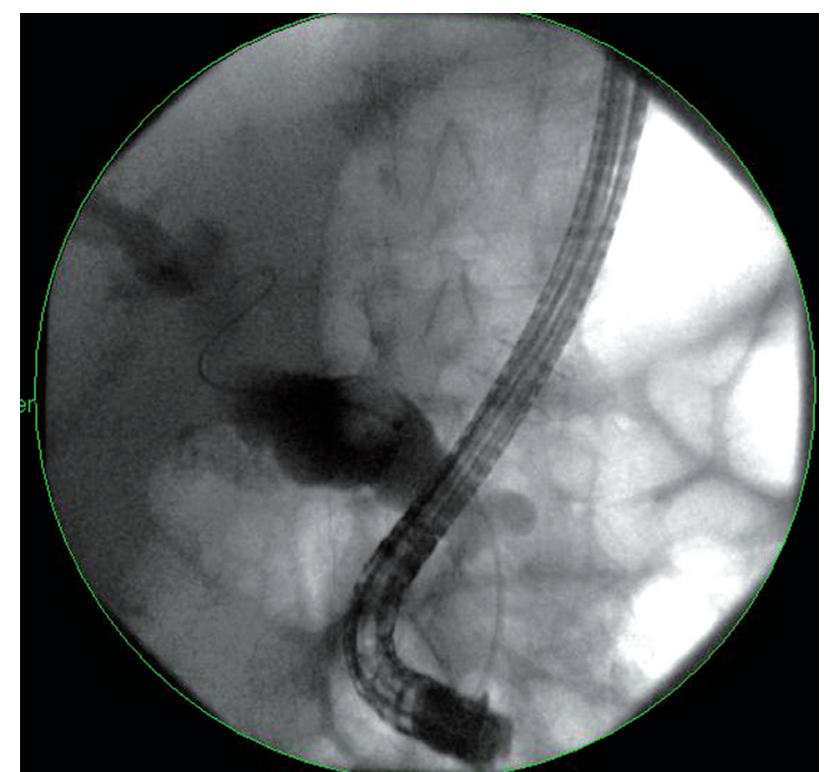

Fig. 6. Fluoroscopic image of endoscopic retrograde cholangiopancreatography through the deployed lumen-apposing metal stent.

the LAMS (Axios; Boston Scientific) delivery system into the excluded stomach. The distal flange of the stent was deployed under fluoroscopic and endosonographic guidance into the excluded stomach, while the proximal flange was deployed under endoscopic visualization into the remnant gastric pouch or afferent limb (Fig. 3). The lumen of the stent was dilated with a dilating balloon to the diameter of the stent (control radial expansion [CRE]; Boston Scientific) (Fig. 4). This allowed for antegrade passage of a duodenoscope (TGF-Q180V; Olympus) or an echoendoscope (GF-UCT180; Olympus) through the LAMS into the stomach remnant and to the ampulla, where conventional ERCP or EUS could be performed, either during the index procedure or during subsequent procedure(s) (Figs, 5, 6). Once ampullary access was no longer required, the LAMS was removed using a snare (25 $\mathrm{mm}$ SnareMaster; Olympus) and the defect was closed using an over-the-scope clip (OTSC; Ovesco, Los Gatos, CA, USA) and/or endoscopic suturing (Overstitch; Apollo Endosurgery, Austin, TX, USA).

\section{RESULTS}

Sixteen patients underwent EDGE (Table 1). The average age of the patients was 55.5 years (range, 7 to 82 ). Thirteen patients $(81 \%)$ were female. Indications for the procedure included biliary stricture $(n=7)$, choledocholithiasis $(n=5)$, recurrent acute pancreatitis $(n=3)$, pancreatic lesion $(n=3)$, and cholangitis $(n=1)$. 
Table 1. Endoscopic Ultrasound (EUS)-Directed Transgastric ERCP and/or EUS ( $n=16)$

\begin{tabular}{ll}
\hline Technical success & $100 \%$ \\
\hline Clinical success & $91 \%(n=10)$ \\
& 5 awaiting fistula maturation prior to ERCP \\
& 1 aborted ERCP \\
& 2.7 days \\
Average hospital stay & $6.25 \%(n=1)$ : jejunal perforation, managed endoscopically \\
Major adverse events & $18.75 \%(n=3)$ \\
Stent dislodgement & -2 repositioned \\
& -1 bridged with FCSEMS \\
& $62.5 \%(n=10):$ removed and closed \\
Stent removal and fistula closure & $25 \%(n=4):$ stent remains for continued access \\
& $6.25 \%(n=1):$ patient passed away from unrelated causes prior to stent removal \\
& $6.25 \%(n=1):$ patient declined further intervention and was lost to follow-up prior to stent removal \\
\hline Average weight change & Negative 2.85 kg \\
\hline
\end{tabular}

ERCP, endoscopic retrograde cholangiopancreatography; EUS, endoscopic ultrasound; FCSEMS, fully covered self-expanding metal stents.

\section{Technical success}

Technical success was achieved in $100 \%$ of patients. Six patients (37.5\%) had gastrogastric fistulas, and $10(62.5 \%)$ had jejunogastric fistulas. All patients had a $15 \mathrm{~mm}$ diameter LAMS successfully placed. The average hospital stay was 2.7 days (range, 0 to 16) postprocedure.

\section{Clinical success}

ERCP and/or EUS through the LAMS was achieved in 10 patients (91\%; 10 of 11 patients). Five patients were still awaiting fistula tract maturation prior to ERCP or EUS at the time of publication, four patients underwent conventional ERCP during the index procedure, six underwent ERCP at a subsequent session following fistula maturation, and one patient had an aborted ERCP due to intestinal perforation, which was managed conservatively. All patients who underwent ERCP experienced successful therapeutic intervention. The average number of ERCP sessions was 3.5. Three patients with pancreatic lesions underwent EUS-fine needle aspiration performed through the LAMS; all three procedures were performed after fistula tract maturation.

\section{Stent removal and fistula closure}

Ten patients (62.5\%) underwent removal of the LAMS. Among these patients, the fistula was closed with endoscopic suturing in seven patients (70\%) and with an OTSC in two patients $(20 \%)$. In one patient, the fistula tract was left to close by secondary intention. Of the remaining six patients, one patient experienced a jejunal perforation during the attempt to advance the duodenoscope through the LAMS. The perforation was closed endoscopically and did not require surgery. One patient passed away from pancreatic cancer prior to LAMS removal, and in four patients, the LAMS was left in place for continued pancreaticobiliary access. Eight of the 10 patients underwent imaging to confirm fistula closure following LAMS removal; among these patients, one had a persistent leak on imaging and was scheduled to undergo repeat closure, while imaging of the remaining seven patients confirmed the absence of any remaining fistula tract. Two patients were awaiting imaging following LAMS removal. No patients required repeat pancreaticobiliary access after LAMS removal.

\section{Adverse events}

In three patients (19\%), the LAMS dislodged during the index procedure. A second LAMS was placed in two of these patients, and a through-the-scope fully covered self-expanding metal stent (FCSEMS; 18×60 mm; Taewoong Medical, Goyang, Korea) was deployed in the third patient without any clinical sequelae. One patient (mentioned above) experienced a jejunal perforation during the attempt to advance the duodenoscope through the jejunogastric LAMS to perform ERCP. The perforation was closed with two adjacent OTSCs without the need for surgical intervention. Three months later, the patient decided to undergo LAMS removal in another institution. There were no instances of bleeding, peritonitis, or pancreatitis in any of the study patients.

\section{Weight change}

The mean weight change from LAMS insertion to removal was negative $2.85 \mathrm{~kg}$. In patients who gained weight, the average gain was $1.7 \mathrm{~kg}$. In patients who lost weight, the average loss was $6.64 \mathrm{~kg}$. 


\section{DISCUSSION}

As bariatric procedures continue to grow in popularity and show effectiveness in reducing obesity and its complications, endoscopists are increasingly required to perform a greater number of interventions in patients with altered anatomy. The development of improved techniques for ERCP in these patients is especially important, as nearly $50 \%$ of postbariatric surgery patients develop gallstones within 2 years. ${ }^{9}$ While there are a number of options for performing ERCP in these patients, including conventional ERCP, EA-ERCP, and LA-ERCP, none of these meet the ideal criteria of being: (1) minimally invasive; (2) able to be performed by a single team; (3) efficacious with a high success rate; and (4) safe with minimal complications. This multicenter experience demonstrates that it is possible to meet all four criteria for an ideal intervention with the use of the EDGE procedure.

It is appropriate to mention that there do exist other fully endoscopic, minimally invasive procedures for accessing the remnant stomach, such as percutaneous-assisted transprosthetic endoscopic therapy (PATENT),${ }^{19}$ EUS-assisted, fluoroscopically guided gastrostomy tube placement, ${ }^{20,21}$ and external EDGE. ${ }^{22,23}$ PATENT consists of advancement of a duodenoscope through an esophageal self-expanding metal stent deployed within a percutaneous gastrostomy tract. While efficacious, the procedure requires deep enteroscopy to access the excluded stomach and the placement of a percutaneous endoscopic gastrostomy (PEG) tube that remains in place for 4 weeks postprocedure. EUS-assisted, fluoroscopically guided gastrostomy tube placement and external EDGE overcome the need for deep enteroscopy by accessing the remnant stomach via EUS, but also require placement of a PEG tube through which conventional ERCP can be performed. While these techniques allow successful, minimally invasive interventions to be performed entirely in the endoscopy suite, they also require PEG tube placement and a minimum of two separate sessions, rendering the techniques unfeasible in patients who require urgent ERCP.

There are notable concerns associated with the utilization of EDGE. The procedure carries a risk of weight gain due to the formation of a gastrogastric or enterogastric fistula, in effect reversing the benefit of the surgical bypass. However, the fistula remains patent for only a short time before closure, and any weight gain would likely be outweighed by the benefit of the procedure. Additionally, in our experience, the mean overall weight change was negative. EDGE may also result in stent dislodgement and potential peritonitis. However, in our experience, no clinical sequelae related to stent dislodgement were observed amongst our patients. Two patients were able to have their LAMS repositioned, and one was bridged with a second FCSEMS. Although one perforation occurred in this series, endoscopic management was possible without the need for surgical intervention, and the patient would likely have undergone successful follow-up ERCP if she had not decided to seek care at another institution.

While the technique is already efficacious, we anticipate that the emergence of novel technological advancements will lead to improvements in the efficacy and overall success of the EDGE procedure. A larger diameter LAMS would facilitate antegrade passage of the duodenoscope through the stent and likely allow for increased rates of ERCP and/or EUS during the same procedure. Similarly, a LAMS containing a cautery feature would negate the need for needle knife dissection and balloon dilation of the fistula tract, and could decrease procedure time and improve efficiency while also improving safety.

Overall, while additional studies are needed to confirm the efficacy and safety of the EDGE procedure, the experience describes here indicates that it is an effective, minimally invasive, single-team, and often single-session solution to the difficulties associated with ERCP in patients who have undergone RYGB.

\section{Conflicts of Interest}

Michel Kahaleh has received grant support from Boston Scientific, Fujinon, EMcison, Xlumena Inc., W.L. Gore, MaunaKea, Apollo Endosurgery, Cook Endoscopy, ASPIRE Bariatrics, GI Dynamics, Olympus, NinePoint Medical, Merit Medical, and MI Tech. He is a consultant for Boston Scientific, Xlumena Inc., Concordia Laboratories, Inc., and MaunaKea Tech.

\section{REFERENCES}

1. Buchwald H, Williams SE. Bariatric surgery worldwide 2003. Obes Surg 2004;14:1157-1164.

2. Buchwald H, Oien DM. Metabolic/bariatric surgery Worldwide 2008. Obes Surg 2009;19:1605-1611.

3. Ribaric G, Buchwald JN, McGlennon TW. Diabetes and weight in comparative studies of bariatric surgery vs conventional medical therapy: a systematic review and meta-analysis. Obes Surg 2014;24:437-455.

4. Ikramuddin S, Korner J, Lee WJ, et al. Roux-en-Y gastric bypass vs intensive medical management for the control of type 2 diabetes, hypertension, and hyperlipidemia: the Diabetes Surgery Study randomized clinical trial. JAMA 2013;309:2240-2249.

5. Sjöström L, Narbro K, Sjöström CD, et al. Effects of bariatric surgery on mortality in Swedish obese subjects. N Engl J Med 2007;357:741-752.

6. Sjöström L, Lindroos AK, Peltonen M, et al. Lifestyle, diabetes, and cardiovascular risk factors 10 years after bariatric surgery. $\mathrm{N} \mathrm{Engl} \mathrm{J} \mathrm{Med}$ 2004;351:2683-2693.

7. Buchwald H, Oien DM. Metabolic/bariatric surgery worldwide 2011. Obes Surg 2013;23:427-436.

8. Hintze RE, Adler A, Veltzke W, Abou-Rebyeh H. Endoscopic access to the papilla of Vater for endoscopic retrograde cholangiopancreatography in patients with billroth II or Roux-en-Y gastrojejunostomy. Endoscopy 1997;29:69-73.

9. Elton E, Hanson BL, Qaseem T, Howell DA. Diagnostic and therapeutic 
ERCP using an enteroscope and a pediatric colonoscope in long-limb surgical bypass patients. Gastrointest Endosc 1998;47:62-67.

10. Skinner M, Popa D, Neumann H, Wilcox CM, Mönkemüller K. ERCP with the overtube-assisted enteroscopy technique: a systematic review. Endoscopy 2014;46:560-572.

11. Lopes TL, Clements RH, Wilcox CM. Laparoscopy-assisted ERCP: experience of a high-volume bariatric surgery center (with video). Gastrointest Endosc 2009;70:1254-1259.

12. Gutierrez JM, Lederer H, Krook JC, Kinney TP, Freeman ML, Jensen EH. Surgical gastrostomy for pancreatobiliary and duodenal access following Roux en Y gastric bypass. J Gastrointest Surg 2009;13:2170-2175.

13. Saleem A, Levy MJ, Petersen BT, Que FG, Baron TH. Laparoscopic assisted ERCP in Roux-en-Y gastric bypass (RYGB) surgery patients. J Gastrointest Surg 2012;16:203-208.

14. Schreiner MA, Chang L, Gluck M, et al. Laparoscopy-assisted versus balloon enteroscopy-assisted ERCP in bariatric post-Roux-en-Y gastric bypass patients. Gastrointest Endosc 2012;75:748-756.

15. Shah RJ, Smolkin M, Yen R, et al. A multicenter, U.S. experience of single-balloon, double-balloon, and rotational overtube-assisted enteroscopy ERCP in patients with surgically altered pancreaticobiliary anatomy (with video). Gastrointest Endosc 2013;77:593-600.

16. Choi EK, Chiorean MV, Coté GA, et al. ERCP via gastrostomy vs. double balloon enteroscopy in patients with prior bariatric Roux-en-Y gastric bypass surgery. Surg Endosc 2013;27:2894-2899.
17. Kedia P, Sharaiha RZ, Kumta NA, Kahaleh M. Internal EUS-directed transgastric ERCP (EDGE): game over. Gastroenterology 2014;147:566568.

18. Kedia P, Tyberg A, Kumta NA, et al. EUS-directed transgastric ERCP for Roux-en-Y gastric bypass anatomy: a minimally invasive approach. Gastrointest Endosc 2015;82:560-565.

19. Law R, Wong Kee Song LM, Petersen BT, Baron TH. Single-session ERCP in patients with previous Roux-en-Y gastric bypass using percutaneous-assisted transprosthetic endoscopic therapy: a case series. Endoscopy 2013;45:671-675.

20. Attam R, Leslie D, Freeman M, Ikramuddin S, Andrade R. EUS-assisted, fluoroscopically guided gastrostomy tube placement in patients with Roux-en-Y gastric bypass: a novel technique for access to the gastric remnant. Gastrointest Endosc 2011;74:677-682.

21. Tekola B, Wang AY, Ramanath M, et al. Percutaneous gastrostomy tube placement to perform transgastrostomy endoscopic retrograde cholangiopancreaticography in patients with Roux-en-Y anatomy. Dig Dis Sci 2011;56:3364-3369.

22. Kedia P, Kumta NA, Widmer J, et al. Endoscopic ultrasound-directed transgastric ERCP (EDGE) for Roux-en-Y anatomy: a novel technique. Endoscopy 2015;47:159-163.

23. Kedia P, Kumta NA, Sharaiha R, Kahaleh M. Bypassing the bypass: EUS-directed transgastric ERCP for Roux-en-Y anatomy. Gastrointest Endosc 2015;81:223-224. 